journal club

\title{
Reduzierte GABA-Konzentration ist mit dem Behinderungsgrad assoziiert
}

Fragestellung: Ist Gamma-Aminobuttersäure (GABA) bei chronisch progredienten Verlaufsformen der Multiplen Sklerose (MS) in besonderem Ausmaß reduziert?

Hintergrund: Neurodegenerative Mechanismen werden als Hauptursache irreversibler Behinderungen bei chronisch progredienter MS betrachtet. Klinische Kernsymptome sind neben Behinderung physischer Aktivitäten vor allem kognitive Defizite. Die Details neurodegenerativer Mechanismen insbesondere als mögliche Folge autoimmunologisch-inflammatorischer Geschehen im ZNS sind noch nicht gut verstanden. GABA ist der wichtigste inhibitorische Botenstoff des ZNS. Inwieweit GABA bei chronisch progredienten Verlaufsformen reduziert ist und mögliche Ansätze für eine Therapie bietet, ist bisher kaum untersucht.

Patienten und Methoden: Bei 30 Patienten mit sekundär progredienter MS und 17 gesunden Kontrollen wurde in einem 3-TeslaMRT mittels Einzel-Voxel MEGA-PRESS (Mescher-Garwood Point resolved spectroscopy)

Cawley N, Solanky BS, Muhlert $\mathrm{N}$ et al. Reduced gamma-aminobutyric acid concentration is associated with physical disability in progressive multiple sclerosis. Brain 2015: 138; 2584-95 der GABA-Gehalt im rechten Hippocampus, präfrontalen Kortex und linken sensomotorischen Kortex bestimmt. Alle Teilnehmer wurden klinisch und neuropsychologisch untersucht. Mittels mul- tipler linearer Regressionsmodelle wurden Unterschiede zwischen Patienten und Kontrollen ermittelt, die geschlechts- und altersspezifisch angepasst wurden. Mittels einfacher Regressionsanalysen wurde die Korrelation zwischen kognitivem Leistungsvermögen sowie physischer Leistungsfähigkeit und GABA-Konzentration der entsprechenden Hirnareale berechnet.

Ergebnisse: Die Patienten waren im Durchschnitt 51 Jahre alt (23 Frauen), hatten einen medianen EDSS von 6,0 und waren im mittel seit vier Jahren chronisch progredient erkrankt. Erwartungsgemäß zeigten Patienten im Vergleich zu gesunden Kontrollen schlechtere physische und kognitive Leistungen. Entsprechend den spezifischen Defiziten zeigten Patienten mit kognitiven Defiziten (vor allem gemessen an der Informationsverarbeitungsgeschwindigkeit und dem sprachlichem Gedächtnis) eine hochspezifische Abnahme der GABA-Konzentration im rechten Hippocampus, während Patienten mit physischen Leistungsdefiziten wiederum eine reduzierte GABA-Konzentration im sensomotorischen Kortex aufwiesen. Die Reduktion der GABAKonzentration korrelierte dabei auch mit dem Ausmaß der Behinderung (z. B. Schwäche des rechten Arms und rechten Beins).

Schlussfolgerungen: Die Reduktion der GABA-Konzentration ist eng mit dem Ausmaß der physischen Behinderung sowie der kognitiven Defizite korreliert und könnte ein wichtiger Marker für frühe neurodegenerative Prozesse sein.

\section{- Kommentar von Volker Limmroth, Köln-Merheim}

\section{GABA-Konzentration als Marker neurodegenerativer Prozesse}

Die Studie sieht auf den ersten Blick wenig spannend aus, denn grundsätzlich würde man davon ausgehen, dass alle wesentlichen Komponenten des zentralen Nervensystems bei Patienten mit chronisch progredenter MS irgendwie betroffen sind. Hier wurden aber nicht offensichtlich betroffene Strukturen MRT-spektroskopisch untersucht (also bereits im regulärem MRT erkennbare Herde), sondern spezifische anatomische Areale mit gut definierten und überprüfbaren Funktionen. Die Areale waren also nicht primär erkennbar betroffen, sondern konnten im regulären MRT normal erscheinen, wiesen aber entsprechend der spezifischen klinischen Defizite eine reduzierte GABA-Konzentration auf. Die Arbeitsgruppe folgert aus dieser Beobachtung, dass eine wesentliche Komponente (früher) neurodegenerativer Prozesse die reduzierte Bildung spezifischer Neurotransmitter wie GABA ist oder die Funktionsbeeinträchtigung der GABA-Rezeptoren. Natürlich steht nicht jedem Neuroradiologen ein 3-Tesla-Gerät zur Verfügung mit dem eine GABA-Spektroskopie durchgeführt werden kann, aber die Arbeit ist ein wichtiger weiterer Schritt in unse- rem Verständnis, wie neurodegenerative Prozesse im Rahmen inflammatorischer Mechanismen entstehen. Darüber hinaus könnte sich die MRT-Spektroskopie langfristig zur Früherkennung chronisch progredienter Verläufe und als objektive Methode zur Verlaufsbeobachtung bei klinischen Studien, in denen Substanzen zur Behandlung chronisch progredienter Verläufe untersucht werden, eignen.

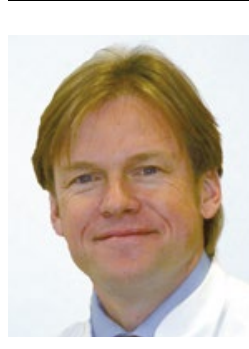

Prof. Dr. med. Volker Limmroth, Köln-Merheim

Chefarzt der Klinik für Neurologie und Palliativmedizin Köln-Merheim E-Mail: LimmrothV@kliniken-koeln.de 